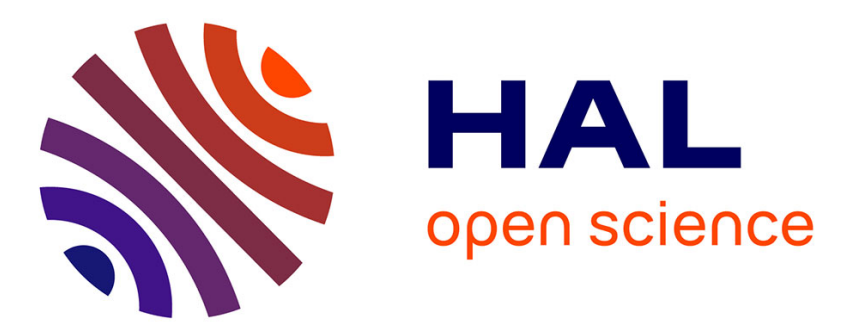

\title{
Le statut et la conservation des archives judiciaires dans l'Orient abbasside (IIe/VIIIe-IVe/Xe siècle) : un réexamen
}

Mathieu Tillier

\section{- To cite this version:}

Mathieu Tillier. Le statut et la conservation des archives judiciaires dans l'Orient abbasside (IIe/VIIIeIVe/Xe siècle): un réexamen. SHMESP. L'autorité de l'écrit au Moyen Âge, Publications de la Sorbonne, pp.263-276, 2009. halshs-00553772

\section{HAL Id: halshs-00553772 \\ https://shs.hal.science/halshs-00553772}

Submitted on 9 Jan 2011

HAL is a multi-disciplinary open access archive for the deposit and dissemination of scientific research documents, whether they are published or not. The documents may come from teaching and research institutions in France or abroad, or from public or private research centers.
L'archive ouverte pluridisciplinaire $\mathbf{H A L}$, est destinée au dépôt et à la diffusion de documents scientifiques de niveau recherche, publiés ou non, émanant des établissements d'enseignement et de recherche français ou étrangers, des laboratoires publics ou privés. 


\title{
Le statut et la conservation des archives judiciaires dans l'Orient abbasside $\left(\mathrm{II}^{\mathrm{e}} / \mathrm{VIII}^{\mathrm{e}}-\mathrm{IV}^{\mathrm{e}} / \mathrm{X}^{\mathrm{e}}\right.$ siècle) $:$ un réexamen
}

\author{
par
}

Mathieu Tillier

Dès l'époque umayyade, les cadis prirent l'habitude de tenir des « archives » $(d \bar{l} w \bar{a} n)$ où étaient conservées les pièces relatives aux procès et aux fonctions administratives de la judicature : des jugements $(\text { sijillāt })^{1}$; des procès-verbaux (mahạdirir) où étaient enregistrées les étapes d'un procès ; des pièces portant la dénomination générale de șukūk et pouvant être des contrats notariés, des promesses, des reconnaissances de dettes, etc. ; diverses listes d'auxiliaires de l'administration judiciaire, de mandataires et de garants, de témoins, de waqf-s, de prisonniers; les lettres rédigées dans le cadre d'une correspondance officielle entre cadis ${ }^{2}$. Si les sources juridiques et narratives attestent l'existence historique de telles pièces et leur archivage, rares sont les documents qui nous sont parvenus avant l'époque ottomane. Quelques papyri d'époque abbasside ont été découverts en Égypte, mais de manière dispersée et décontextualisée ${ }^{3}$, ce qui ne permet pas de reconstituer leur appartenance originelle aux archives officielles d'un cadi. À notre connaissance, seuls les documents mamlūks du Haram al-Sharīf, récemment étudiés par Christian Müller, peuvent être considérés comme un ensemble cohérent d'archives judiciaires médiévales ${ }^{4}$.

La rareté des documents judiciaires datant du Moyen Âge, conjuguée au manque d'informations disponibles sur les anciens modes d'archivage, a conduit certains chercheurs à mettre en doute l'idée même d'une conservation archivistique sur le long terme par l'institution judiciaire. Dans un article de 1998, Wael B. Hallaq met en particulier l'accent sur l'absence de préservation pérenne d'une telle documentation. Analysant la transmission des archives d'un cadi à son successeur, il conclut que celles-ci

\footnotetext{
${ }^{1}$ Sur la forme de leur enregistrement, voir Y. RĀĞIB, Actes de vente d'esclaves et d'animaux d'Égypte médiévale, Le Caire, 2006, t. 2, p. 118.

${ }^{2}$ Pour le détail de ces pièces, voir B. JOHANSEN, «Formes de langage et fonctions publiques : stéréotypes, témoins et offices dans la preuve par l'écrit en droit musulman », Arabica, 44 (1997), p. 344-348 ; W.B. HALlAQ, « The qāậl s dīwān (sijill) before the Ottomans », Bulletin of the School of Oriental and African Studies, 61 (1998), p. 419-421 ; P.M. SIJPESTEIJN, « The Archival Mind in Early Islamic Egypt: Two Arabic Papyri », From al-Andalus to Khurasan, éd. P.M. SIJPESTEIJN et alii, Leyde-Boston, 2007, p. 176-177. Sur les pratiques d'archivage étatique, voir M.M. BravmanN, «The State Archives in the Early Islamic Era », Arabica, 15 (1968), p. 87-89.

${ }^{3}$ Y. RĀĠIB, «Les plus anciens papyrus arabes », Annales Islamologiques, 30 (1996), p. 2.

${ }^{4}$ Cf. L.S. Northrup et A.A. AbUL-HAJJ, «A Collection of Medieval Arabic Documents in the Islamic Museum at the Haram al-Šarif », Arabica, 25 (1978), p. 283.
} 
demeuraient en possession de l'ancien cadi. Sortant de la sphère institutionnelle pour pénétrer dans celle du privé, ces archives principalement composées de feuillets volants auraient fini par être réutilisées ou détruites ${ }^{5}$. Nous proposons de réexaminer ici cette hypothèse en nous concentrant sur l'Irak entre le VIII ${ }^{\mathrm{e}}$ et le $\mathrm{X}^{\mathrm{e}}$ siècle : il nous semble en effet que plusieurs indices jusqu'ici négligés plaident au contraire en faveur de la conservation du dīwān d'un cadi par l'institution judiciaire. Il n'en demeure pas moins que le statut juridique ambigu des archives rendait leur utilisation malaisée ; il faudra donc prendre en considération la place et la valeur de l'écrit dans la procédure judiciaire, en nous penchant tout particulièrement sur la littérature juridique relative aux waqf-s.

\section{Transmission des archives et inventaires}

La littérature juridique est le principal témoin de la tenue d'archives judiciaires. Les premiers ouvrages de disciplina judicis (adab al-qūd $\bar{\imath}$ ) décrivent avec précision la procédure de transmission de ces archives d'un cadi à son successeur: la rotation fréquente des juges - au gré des nominations et des révocations par le pouvoir - nécessitait en effet la remise en bonne et due forme du dīwān, garant essentiel de la continuité judiciaire au sein d'une circonscription ${ }^{6}$. Cette transmission est décrite pour la première fois par le juriste hanafite al-Khașșāf (m. 261/874) dans son Adab al-qā $\bar{c} \bar{\imath}$ :

Lorsque le cadi veut prendre possession des archives (dīwān) du cadi précédent, il envoie deux de ses hommes de confiance (rajulayn min thiqāti-hi) se les faire remettre. [... $]^{7} \mathrm{Si}$ [le cadi] veut en prendre possession, [ses deux hommes de confiance] doivent examiner les [enregistrements] d'aveux, les témoignages et autres procès-verbaux (mahā dir) qu'elles comportent: ils prennent les caisses (qamātirir) présentes dans [ces archives], cachetées, [et font l'inventaire] des jugements (sijillāt), des reconnaissances de dette (șikāk bi-lamwāl 'alā l-nās) et des autres documents juridiques $(\text { hujaj } l i-l-n \overline{a s})^{8}$. Ils écrivent ce qu'ils y trouvent au fur et à mesure, disant «Parmi cela, une caisse comportant la copie d'un jugement en faveur d'Untel fils d'Untel, [lui accordant] telle et telle chose ; la copie d'un jugement en faveur d'Untel fils d'Untel, [lui accordant] telle et telle chose, etc. », et finissent par écrire le nombre total de

\footnotetext{
${ }^{5}$ HallaQ, «The qā $\bar{d} \vec{\imath} \mathrm{s} d \bar{\imath} w \bar{a} n »$, loc. cit., p. 426, 435.

${ }^{6}$ Ibid., p. 425.

${ }^{7}$ Al-Jașsaāṣ, le commentateur d'al-Khașșāf, intervient ici pour justifier l'utilisation de deux hommes de confiance : «Ils doivent être au nombre de deux - de sexe masculin -, afin que [la procédure] ne puisse prêter le flanc à aucune accusation, et pour que les affaires et les droits de la population soient préservés. »

${ }^{8} \mathrm{Au} \mathrm{V} \mathrm{V}^{\mathrm{e}} / \mathrm{XI} \mathrm{e}^{\mathrm{e}}$ siècle, al-Māwardī explique que le $d \bar{l} w \bar{a} n$ contient les pièces (hujaj) rédigées pour prouver les droits des plaideurs. Elles sont copiées en deux exemplaires, dont l'un est remis à l'intéressé. Al-Māwardī, Adab al-qāộ̀, Bagdad, 1971, t. 1, p. 220.
} 
documents trouvés. Tout ceci doit se faire en présence du cadi révoqué ou de deux de ses administrateurs (aminayn min umanā' $i$-hi $)^{9}$.

En admettant que la procédure décrite était appliquée dans les faits, le cadi révoqué - pour peu qu'il fût encore en vie - y jouait son dernier rôle décisif : la transmission du dīwān tenait lieu de passation de pouvoir à son successeur. Il était le meilleur connaisseur des dossiers archivés, tout particulièrement ceux qu'il avait lui-même traités au cours de son mandat, et en garantissait le contenu et la véracité. Al-Khașạâf précise un peu plus loin que «[les deux hommes de confiance] interrogent l'[ancien] cadi au fur et à mesure sur ce qu'ils trouvent, et en prennent possession en écrivant les explications qu'il donne au sujet [de chaque pièce]. » Les caisses enfermant les archives étaient soigneusement ouvertes par les auxiliaires du cadi entrant : ils en vérifiaient le contenu et dressaient un inventaire écrit de ce qu'ils trouvaient, établissant de la sorte une liste détaillée des documents en possession de l'ancien cadi. Un état des lieux complet des affaires judiciaires de chaque district était donc en principe réalisé à chaque révocation et nouvelle nomination.

La description de cette procédure laisse néanmoins une zone d'ombre : celle du devenir des archives sur le long terme. Pour sa part, Hallaq suppose que seul l'inventaire des documents était conservé par le nouveau cadi lors de cette passation de pouvoirs, tandis que les archives originales demeuraient aux mains du cadi révoqué : cela expliquerait que celles-ci, échappant à une conservation par l'institution judiciaire, aient fini par disparaître ${ }^{10}$. Hallaq s'appuie tout spécialement sur l'exemple de la transition entre deux cadis bașriens, en 166/782-83, rapporté par Wakī dans un passage équivoque de ses Akhbār al-quḍ̄at ${ }^{11}$ :

'Abd Allāh b. al-Ḥasan al-Mu'addib nous rapporta d'après al-Numayrī, d'après Khālid b. 'Abd al-'Azīz :

Je vis Khālid b. Țalīq, le jour où il [se mit à] siéger pour rendre la justice, à son arrivée de Bagdad, s'asseoir dans la cour de la mosquée, près du bassin. Il convoqua 'Ubayd Allāh b. al-Hasan et on l'amena. Il ordonna à son héraut de crier : "Où est 'Ubayd Allāh b. al-Hasan ? », et la foule lança des imprécations contre Hālid. Lorsqu'il s'assit devant lui, il dit : «Voici les documents (kutub); qui en prend réception? Avant moi, il était d'usage de les remettre [au nouveau cadi] (yusallimūna-hā). Mais j'ai décidé d'en établir deux copies/exemplaires (nuskhatayn) en présence de témoins honorables : tu en prendra une, j'en aurai une [autre], et je serai tenu par cela ('alayya gharāma dhālika). Envoie des témoins honorables et des scribes qui t'agréent!» Puis il se leva et la foule appela la bénédiction [de Dieu] sur lui. Les documents furent copiés en deux

${ }^{9}$ Al-KHAșșĀF, Adab al-qāộ̉, Le Caire, 1978, p. 57

${ }^{10}$ HallaQ, « The qāạd $\mathrm{s} d \bar{\imath} w \bar{a} n »$, loc. cit., p. 435.

${ }^{11}$ Ibid., p. 426. 
exemplaires afin qu'il ne change rien à ses jugements ${ }^{12}$.

Ce passage est interprété de façon contestable par Hallaq, qui considère Khālid b. Talīq comme le premier cadi à avoir insisté pour conserver le dìwān original, tandis que le nouveau en faisait établir une double copie ${ }^{13}$. Il est clair, cependant, que le cadi sortant n'est pas Khālid b. Țalīq, mais 'Ubayd Allāh b. al-Hasan al-'Anbarī. Wakī' ne précise pas, par ailleurs, lequel des deux hommes conserve les archives originales et lequel reçoit la copie. Malgré l'ambiguïté des pronoms personnels, la structure du texte suggère enfin que ce n'est pas 'Ubayd Allāh, l'ancien cadi, qui insiste pour conserver ses archives, mais que Khālid propose de lui remettre un exemplaire des documents afin que 'Ubayd Allāh puisse vérifier qu'il respectera ses jugements. Le récit est en effet centré sur le retournement de l'opinion publique en faveur de Khālid $b$. Ṭalīq : conspué dans un premier temps, il obtient les louanges de la foule grâce à cette initiative. Rien ne permet d'affirmer que cette pratique ait été conservée par la suite: le prestige spécifique dont 'Ubayd Allāh b. al-Hasan jouissait auprès des Bașriens ${ }^{14}$ plaide au contraire en faveur du caractère exceptionnel de cette mesure. Le cadi entrant devait reconnaître l'autorité de 'Ubayd Allāh sous peine de pâtir d'une réputation inversement proportionnelle à la popularité de son prédécesseur ${ }^{15}$.

La terminologie employée pour décrire la transmission du dīwān à un nouveau cadi suggère pour sa part qu'un transfert intégral des documents avait lieu. Dans ce récit, le verbe sallama ou tasallama (remettre, se faire remettre) semble en témoigner ${ }^{16}$; les juristes al-Khașșāf ou, plus tard, Ibn Māza, emploient le verbe qabậda (saisir), indiquant par là que le nouveau cadi prenait physiquement possession des archives avant de les trier par types de documents ${ }^{17}$. La littérature biographique suggère également un transfert physique du dīwān d'un cadi à un autre ${ }^{18}$. $\mathrm{Au} \mathrm{IV}^{\mathrm{e}} / \mathrm{X}^{\mathrm{e}}$ siècle, à Fustật, un cadi entrant s'empara même des archives de son prédécesseur en remplaçant son sceau par le $\operatorname{sien}^{19}$. Reste à savoir comment le dīwān d'un cadi était gardé et dans quelle mesure les documents y étaient conservés de façon pérenne.

\footnotetext{
${ }^{12} \mathrm{WAKI}^{`}$, Akhbār al-quḍāt, Le Caire, 1947-50, t. 2, p. 125.

${ }^{13}$ HALLAQ, «The qā $\bar{d} \vec{\imath}$ s $d \bar{l} w \bar{a} n »$, loc. cit., p. 426.

${ }^{14}$ Voir M. TiLlieR, «Un traité politique du $\mathrm{II}^{\mathrm{e}} / \mathrm{VIII}$ e siècle. L'épître de 'Ubayd Allāh b. alHasan al- 'Anbarī au calife al-Mahdī », Annales Islamologiques, 40 (2006), p. 144.

${ }^{15} \mathrm{D}$ 'autres récits évoquent la transmission des archives à un nouveau cadi. Voir notamment IBN ḤAĞAR, Raf ‘ al-iṣr, Le Caire, 1998, p. 420 (trad. M. TILliER, Vies des cadis de Miṣr, Le Caire, 2002, p. 124).

${ }^{16}$ Voir également AL-MĀWARDĪ, Adab al-qạ̣̄̂, t. 1, p. 220.

${ }^{17}$ IBn MĀZA, Sharh adab al-qāḍ̄, Beyrouth, 1994, p. 38.

${ }_{18}^{18}$ IBn Hajar, Raf' al-ișr, p. 179, 183, 328 (trad. TILLIER, Vies, op. cit., p. 113, 151, 177).

${ }^{19}$ IBN ḤAJAR, Raf' al-iṣr, p. 185 (trad. TILLIER, Vies, op. cit., p. 154).
} 


\section{La conservation des archives}

\subsection{Une préservation institutionnelle}

La théorie de Hallaq repose en partie sur la difficulté à identifier, à travers les sources, des modes de conservation institutionnelle des archives. Il est vrai qu'à l'époque abbasside, aucun local public ne semblait réservé à l'archivage des documents judiciaires. En Égypte, le dīwān fut longtemps gardé en dépôt chez un particulier ${ }^{20}$. Vers $212 / 827$, le cadi 'İsā b. alMunkadir louait une maison pour les entreposer et en faisait chaque soir sceller la porte ${ }^{21}$. Dans les villes du Sud irakien, les cadis conservaient chez eux leur qimatr, sorte de caisse à archives dont l'usage se répandit d'abord en Irak, puis en Égypte ${ }^{22}$ : au début du $\mathrm{III}^{\mathrm{e}} / \mathrm{IX}^{\mathrm{e}}$ siècle, ils l'apportaient parfois eux-mêmes à l'audience ${ }^{23}$ et cette pratique semblait érigée en règle un siècle plus $\operatorname{tard}^{24}$.

La mise en dépôt du dīwān chez un ou plusieurs particuliers ne signifiait pas pour autant que les archives étaient privatisées, ni que les cadis révoqués emportaient les documents établis au cours de leurs mandats. Si aucun local public n'était affecté à leur conservation, les dépositaires ne les conservaient pas moins au nom de l'institution judiciaire pour laquelle ils exerçaient une fonction officielle. Al-Kindī raconte à propos d'al-'Umarī (en poste de $185 / 801$ à 194/810) qu'un faux document fut introduit parmi les archives d'un précédent cadi de Fustạaț, afin de faire croire qu'il avait déjà établi (ithbāt) la généalogie d'un groupe à l'origine contestée. Le texte mentionne un mutawallī de ce dīwān, un "préposé » responsable de sa conservation qui n'était visiblement ni ledit cadi, ni de sa descendance, et à qui il fallut verser un pot-de-vin de mille dinars pour qu'il ferme les yeux sur la fraude ${ }^{25}$. Ce préposé gardait visiblement le dīwān du seul cadi al-Mufaḍdal b. Faḍāla, ce qui laisse penser que d'autres agissaient de même pour ceux d'autres cadis. Si ce mode de conservation impliquait bien, semble-t-il, une dispersion des archives, celles-ci demeuraient publiques et l'on pouvait couramment aller consulter celles d'un cadi antérieur afin de vérifier ou d'établir des faits. Le

\footnotetext{
${ }^{20}$ E. Tyan (Histoire de l'organisation judiciaire en pays d'Islam, Leyde, 1960, p. 253) s'appuie sur al-Kindī pour avancer que les archives étaient gardées au domicile du greffier. Selon al-Kindī, au début de la judicature de 'Īsā b. al-Munkadir, le qimațr était conservé dans une «boutique » (hānūt) située dans la maison de 'Amr b. Khālid (AL-KINDĪ, Akhbār quḍāt Mișr, Leyde, 1912, p. 437). Si ce dernier personnage fut effectivement secrétaire de plusieurs cadis, il n'est néanmoins pas cité dans la liste des greffiers d'Ibn al-Munkadir proposée par alKindī (Akhbār qudāat Miṣr, p. 435-36).

${ }^{21}$ AL-KINDİ, Ahbār qud̄àt Mișr, p. 437.

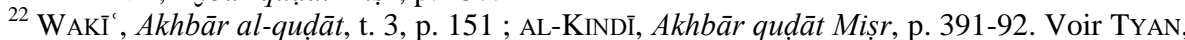
Organisation judiciaire, op. cit., p. 253 ; 'I.M. SHABĀRŪ, al-Qaḍ̄' wa-l-qud̄āt fì-l-islām, Beyrouth, 1983, p. 63 ; HALLAQ, « The qā $\bar{\imath} \vec{\imath} \mathrm{s} d \bar{\imath} w \bar{a} n », l o c$. cit., p. 433.

${ }^{23} \mathrm{WAK \overline {I }}^{\prime}$, Akhbār al-qud̄āt, t. 2, p. 109, 164.

${ }^{24}$ IBN AL-QĀȘS Adab al-qā

${ }^{25}$ AL-KINDĪ, Akhbār quẹ̄āt Miṣr, p. 398.
} 
cadi dudit dīwān avait été définitivement révoqué de la judicature en $177 / 793^{26}$, une dizaine d'années avant le procès qui donna lieu à cette falsification. Entre temps, deux cadis avaient successivement exercé la judicature de Fustâtạ. À moyen terme donc, les archives des anciens cadis demeuraient une documentation publique. Au milieu du IV $/ \mathrm{X}^{\mathrm{e}}$ siècle, à Bagdad, le dīwān était également placé sous la surveillance d'un auxiliaire spécifique, portant le titre de «gardien des archives judiciaires»(khāzin dīwān al-hukm) ${ }^{27}$.

D'autres récits montrent que les cadis avaient toujours accès aux documents archivés par leurs prédécesseurs. À son arrivée en poste à alBașra, au $\mathrm{III}^{\mathrm{e}} / \mathrm{IX}^{\mathrm{e}}$ siècle, le cadi al-Ḥasan b. 'Ubayd Allāh al-'Anbarī hérita d'archives concernant des individus résidant désormais à Bagdad. Il semble même que certains validèrent à nouveau leurs actes devant le nouveau cadi en apportant la preuve testimoniale (bayyina) de leur bien-fondé. Pour des raisons visiblement politiques, le grand cadi Ibn Abī Du'ād réclama le transfert de ces archives mais le cadi refusa, arguant de son devoir de protéger les ayants droit. Mais face à l'entrée en scène du calife, il écrivit à ce dernier que « les archives [étaient] celles du Commandeur des croyants », qui pouvait donc en disposer comme il l'entendait ${ }^{28}$. Il exprimait par là que la préservation d'anciennes archives relevait toujours de l'État et de son souverain, le calife.

La prise de possession du dīwān par un cadi entrant ne signifiait pas toujours que les archives judiciaires s'additionnaient les unes aux autres, au fil des désignations, dans le cadre d'un corpus archivistique unique et cohérent. D'un côté, l'exemple égyptien plaide en faveur d'une certaine dispersion des archives, le dīwān de chaque cadi étant confié à un responsable individuel. D'un autre côté, celui de Bașra suggère que certains cadis intégraient au contraire les archives de leurs prédécesseurs à leurs propres $d \bar{\imath} w \bar{a} n$-s et que la valeur juridique de certaines pièces était réactivée par le biais de bayyina-s. Peut-être faut-il voir ici des pratiques régionalement différenciées. Quoi qu'il en soit, l'hypothèse d'une privatisation d'archives devenues obsolètes ne semble pas fondée : dans les deux cas, leur conservation semblait bien relever de l'institution judiciaire et, au niveau supérieur, du califat.

\subsection{Fonction des inventaires}

Il faut donc dissocier le dīwān de l'ancien cadi, en tant que tel, de son inventaire au moment de la passation de pouvoir. Un cadi entrant continuait, si nécessaire, à consulter les archives de ses prédécesseurs et le catalogue dressé à son arrivée en poste ne se substituait pas purement et simplement à

${ }^{26}$ AL-KINDĪ, Akhbār quḍāt Mișr, p. 387.

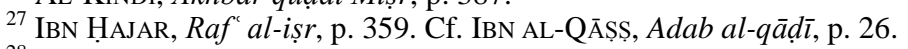

${ }^{28} \mathrm{WAK \overline {I }}^{\prime}$, Akhbār al-quạāt, t. 2, p. 174. 
ces archives. La copie exceptionnelle dont fit l'objet le dīwān du cadi bașrien 'Ubayd Allāh b. al-Hasan ne doit pas non plus être confondue avec l'établissement régulier de tels inventaires selon la doctrine hanafite. Quelle était leur fonction? Il est clair, comme le souligne Hallaq ${ }^{29}$, qu'ils devaient tout d'abord fournir au cadi un outil de référence, permettant de retrouver rapidement les cas auparavant traités par l'administration judiciaire, avant d'aller éventuellement consulter les archives originales. Mais il faut aussi penser qu'un tel répertoire notarié évitait l'introduction postérieure de faux documents dans les archives ou prévenait la disparition de certaines pièces : un document ne figurant pas dans l'état des lieux rédigé au moment de la transition entre deux cadis devait être considéré avec suspicion. C'est bien parce que le cadi accédait toujours aux archives de ses prédécesseurs que de tels inventaires - fixant définitivement leur contenu au moment de leur clôture - étaient indispensables.

\section{Statut juridique du $d \bar{i} w \bar{a} n$}

\subsection{Présomptions judiciaires}

Si l'hypothèse d'une privatisation des archives ne nous semble pas convaincante, celle d'une conservation peu rigoureuse sur le moyen ou le long terme mérite de retenir toute notre attention. En effet l'utilisation d'un ancien dìwān était limitée par la valeur probatoire reconnue aux pièces archivées. Les preuves légales acceptées en justices se limitaient en règle générale à quatre catégories : 1. La bayyina, témoignage de deux hommes honorables, était considérée comme la preuve par excellence ; elle incombait en principe au demandeur. 2. Si le demandeur ne pouvait produire de preuve testimoniale, le cadi pouvait demander au défendeur de prêter serment (yamīn) afin de s'innocenter. 3. Le refus de prêter serment (al-nukūl 'an alyaminn) était également considéré comme une preuve de la culpabilité de celui à qui il était déféré. 4. L'aveu du défendeur possédait enfin une valeur probatoire $^{30}$. Les historiens ont depuis longtemps souligné la nature essentiellement orale de ces preuves. L'écrit pouvait aussi se voir accorder une valeur probatoire, mais à condition que le document produit soit notarié : le témoignage écrit - puis oral en cas de procès - de personnes honorables permettait seul d'intégrer le document dans le cadre légal de la bayyina ${ }^{31}$. Néanmoins Baber Johansen a montré que les archives d'un cadi n'avaient plus valeur de preuve après sa mort ou sa révocation. Pour les hanafites en particulier, seul le cadi ayant instruit les affaires concernées par ces documents pouvait les considérer comme des preuves suffisantes : ils ne

\footnotetext{
${ }^{29}$ HallaQ, « The qā

30 Voir R. Brunschvig, «Le système de la preuve en droit musulman », Études d'Islamologie, Paris, 1976, t. 2, p. 208 sq.

${ }^{31}$ J.A. WAKIN, The Function of Documents in Islamic Law, Albany, 1972, p. 7.
} 
pouvaient « remplacer la mémoire individuelle du juge » que sur une durée limitée, tant que celui-ci était en fonctions ${ }^{32}$. Simple aide-mémoire, les archives ne permettaient pas d'assurer une parfaite transition judiciaire. Les procès interrompus par la mort ou la révocation d'un cadi devaient en grande partie être recommencés par son successeur: les documents de thubūt, enregistrant par écrit les témoignages et les aveux avant le rendu du jugement, ne pouvaient être pris en considération par le cadi entrant. Celui-ci devait à nouveau entendre ces preuves, établir de nouveaux documents de thubüt, avant de pouvoir enfin rendre son jugement. Cela signifie que, malgré l'existence d'archives, un procès inachevé était annulé et devait être entièrement repris. De même, si un jugement avait été rendu par le cadi précédent mais n'avait pas encore été exécuté, le sijill portant enregistrement du jugement ne suffisait pas pour mener la procédure à son terme : il fallait soit que la culpabilité du prévenu soit de nouveau prouvée - notamment en cas d'emprisonnement d'un débiteur insolvable - soit, éventuellement, que le jugement du cadi précédent soit confirmé par une bayyina orale $^{33}$.

Compte tenu de sa faible valeur probatoire, il serait logique, comme le suppose Hallaq, que le dīwān d'un ancien cadi sorte de la sphère publique et disparaisse rapidement. Or nous avons vu que tel n'était pas le cas et qu'alKhașạâf insistait au contraire sur une transmission méticuleuse des archives au cadi entrant et sur leur importance pour le respect de la continuité judiciaire. Il y a là un paradoxe qui ne peut être résolu qu'au prix d'un réexamen du statut de ces archives. Une piste a jusqu'ici été peu explorée : celle des présomptions judiciaires. La continuité entre deux cadis n'était pas uniquement assurée par la production de preuves de l'activité judiciaire passée : en certains cas, l'absence de preuves obligeait un nouveau cadi à présumer la véracité des faits. Par exemple, al-Khașșāf explique qu'à son arrivée en poste, un cadi peut trouver des prisonniers dont personne ne peut prouver la culpabilité. Malgré cela, il ne doit pas les libérer sur-le-champ : son prédécesseur doit a priori être considéré comme juste, et les incarcérations comme fondées ${ }^{34}$. Le détenu est présumé coupable et ce n'est qu'au bout d'un temps suffisant pour que son accusateur ait pu se présenter que le cadi le libère sous caution ${ }^{35}$. Si les archives anciennes n'avaient pas valeur de preuves, elles semblaient au moins avoir celle de présomptions : traces des activités judiciaires passées, elles témoignaient que des faits avaient été établis, que des preuves avaient été produites, qu'un jugement avait été rendu. En tant que telles, elles constituaient des indices suffisants

\footnotetext{
32 JOHANSEN, «Formes de langage », loc. cit., p. 333-376.

${ }^{33}$ M. TILLIER, Les cadis d'Iraq à l'époque 'abbāside : organisation administrative et rapports au pouvoir (132/750-334/945), Thèse de doctorat, Lyon, 2004, p. 367 sq.

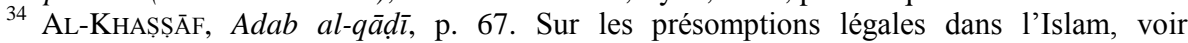
J. SCHACHT, Introduction au droit musulman, Paris, 1983, p. 159 ; BRUNSCHVIG, « Le système de la preuve », loc. cit., p. 207.

${ }^{35}$ Al-KHAȘȘĀF, Adab al-qā ḍ̄, p. 64.
} 
pour orienter la compréhension et le travail du nouveau cadi. Cette hypothèse semble confirmée par al-Shāfi 'î : il permet à celui-ci d' "alléger » (yukhaffif) la procédure lors de la réouverture d'un dossier non mené à terme par son prédécesseur ${ }^{36}$. Armé des fortes présomptions que constituaient les archives, le cadi pouvait reprendre le procès de manière accélérée et parvenir rapidement à un jugement. De même, le sijill enregistrant un jugement non appliqué de son prédécesseur portait en principe les noms des témoins susceptibles de l'attester: le nouveau cadi pouvait ainsi aisément les rechercher et les faire témoigner du jugement.

\subsection{Une exception? Waqf-s et valeur probatoire de l'écrit}

Les présomptions établies grâce au dīwān d'un ancien cadi dépassaient ce simple statut par l'adjonction de preuves légales orales. Cela signifie néanmoins que leur valeur était théoriquement limitée dans le temps, à l'échelle d'une vie humaine: une fois les derniers témoins de l'affaire disparus, il devenait impossible de prouver la validité des documents. Sur ce point au moins, l'hypothèse de Hallaq semblerait justifiée : au bout de quelques décennies, nombre d'archives pouvaient perdre toute valeur juridique et se voir mises au rebut. Pourtant, certaines affaires étaient appelées à se prolonger sur plusieurs générations : les revenus des waqf-s, notamment, pouvaient faire l'objet de procès entre bénéficiaires longtemps après leurs fondations ${ }^{37}$. Afin de trancher de telles disputes, il était nécessaire de revenir aux instructions du fondateur. Or d'anciens waqf-s pouvaient ne pas avoir fait l'objet d'enregistrement écrit auprès du cadi, ou plus aucun témoin ne pouvait attester leurs stipulations. La gestion des biens de mainmorte était suffisamment délicate au $\mathrm{III}^{\mathrm{e}} / \mathrm{IX}^{\mathrm{e}}$ siècle pour que deux auteurs hanafites, Hilāl al-Ra'y et al-Khașșāf, y dédient chacun un ouvrage détaille $^{3 \dot{8}}$. Nous proposons d'explorer plus spécifiquement le second, afin de voir comment le juriste envisageait l'utilisation des archives dans la gestion des waqf-s.

Les actes de fondations en waqf-s font partie des principales archives islamiques ayant survécu pour des périodes plus tardives. Il est généralement admis que de tels documents furent définitivement intégrés aux dīwān-s des cadis vers la fin du $\mathrm{II}^{\mathrm{e}} / \mathrm{VIII}^{\mathrm{e}}$ siècle $^{39}$. Les Ahkām al-awqāf d'al-Khașṣāf confirment qu'à l'époque de leur rédaction - vers le milieu du siècle suivant

\footnotetext{
${ }^{36}$ AL-SHĀFI' '̇, Kitāb al-umm, Beyrouth, 1973, t. 6, p. 204.

${ }^{37}$ Voir par exemple IBN HAJAR, Raf ' al-ișr, p. 122-23 (trad. Tillier, Vies, op. cit., p. 47-50).

${ }^{38}$ Claude Cahen s'est en particulier appuyé sur eux dans son article « Réflexions sur le waqf ancien », Studia Islamica, 14 (1961), p. 37-56, rééd. dans ID., Les peuples musulmans dans l'histoire médiévale, Damas, 1977, p. 287-306. Voir également P.C. HenNigan, The Birth of a Legal Institution. The Formation of the Waqf in Third-Century A.H. Hanafi Legal Discourse, Leyde-Boston, 2004.

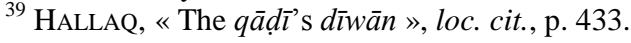


-, l'écrit était largement utilisé pour la fondation d'un bien de mainmorte : le juriste hanafite évoque à de nombreuses reprises le kitāb (écrit, document) du waqf $^{40}$, sa 'uqda (pacte, sous-entendu écrit) ${ }^{41}$, ou les shurūt (conditions) stipulées par le fondateur - sous-entendu dans un acte écrit ${ }^{42}$. Deux inscriptions datées de 300 et 301/911-13, à al-Ramla, mentionnent également le kitāb de fondation d'un waqf $f^{43}$. Il ne faudrait néanmoins pas en conclure trop hâtivement que l'établissement d'un acte écrit était déjà systématique. Al-Khașșāf mentionne en effet à plusieurs reprises le cas où un cadi rechercherait sans succès dans son dīwān ou celui de ses prédécesseurs l'acte de fondation d'un waqf afin de trancher un litige ${ }^{44}$ : il était donc envisageable à son époque qu'une propriété ait été immobilisée sans passage devant un cadi. Peut-être cette situation était-elle particulièrement courante en milieu rural, à distance du tribunal. Malgré l'une des inscriptions d'alRamla, qui concerne une terre agricole ${ }^{45}$, les développements qu'al-Khașșâf consacre aux terres immobilisées dans les campagnes ne font nulle référence à des documents écrits: les types de procès considérés reposent exclusivement sur des témoignages oraux, des aveux et des serments ${ }^{46}$.

Néanmoins il est probable qu'au milieu $\mathrm{du} \mathrm{III}^{\mathrm{e}} / \mathrm{IX}^{\mathrm{e}}$ siècle, nombre de $w a q f$-s étaient enregistrés auprès du tribunal et que leurs actes de fondation étaient archivés dans le dīwān. La fonction qu'al-Khașșāf attribue à de tels documents est particulièrement intéressante : contrairement à la doctrine générale, il va en effet jusqu'à leur reconnaître une valeur probatoire en l'absence de témoignages complémentaires. À plusieurs reprises, il évoque les «très anciens waqf-s» (al-wuqū f al-mutaqādima) et se demande comment trancher des conflits relatifs à la distribution de leurs revenus lorsque tous les témoins de la transformation en bien de mainmorte ont disparu. Si le cadi a la gestion du bien ( $f \grave{i}$ aydī $l$-qã $d \hat{l})$ et si les règles (rusūm) le régissant sont enregistrées dans les archives judiciaires (dawāwìn al$q u d \underline{a} t)^{47}$, le cadi doit s'y conformer sans autre preuve : conscient de déroger à la norme relative à la valeur probatoire des archives, al-Khașșâf recourt au principe d'istihsān, lui permettant de «préférer» une solution ponctuellement meilleure que celle découlant directement de la règle

\footnotetext{
${ }^{40}$ AL-KHAȘȘĀF, Ahkām al-awqāff, Beyrouth, 1999, p. 102, 132, 224, 232, 241, 252, 290.

${ }^{41}$ Ibid., p. 51.

${ }^{42}$ Ibid., p. 74.

${ }^{43}$ M. Sharon, "A Waqf Inscription from Ramlah », Arabica, 13 (1966), p. 77, 83 ; ID., «Waqf Inscription from Ramla c. 300/912-13», Bulletin of the School of Oriental and African Studies, 60 (1997), p. 100.

${ }_{44}$ AL-KHAȘȘĀF, Ahkām al-awqā f, p. 114, 159. À la même époque, en Égypte, certains biens de mainmorte n'étaient pas encore enregistrés dans le dīwān du cadi Ibn Abī l-Layth. ALKINDĪ, Akhbār qudāat Miṣr, p. 450.

${ }^{45}$ M. SHARON, «Waqf Inscription from Ramla c. 300/912-13 », loc. cit., p. 101.

${ }^{46}$ AL-KhașșāF, Ahkām al-awqājf, p. 162 sq.

${ }^{47}$ L'emploi du pluriel semble ici confirmer la dispersion des documents dans les dīwān-s individuels de chaque ancien cadi.
} 
générale $^{48}$. En l'absence de témoins, donc, l'acte de fondation pouvait servir de preuve. Un cadi nouvellement nommé dans une ville pouvait également se référer aux rusūm et aux listes d'administrateurs (umanā') trouvés dans le dīwān de son prédécesseur ${ }^{49}$. En cas de litige, les actes de waqf conservés dans le dīwān avaient plus de poids aux yeux du cadi que la parole des héritiers de l'ancien fondateur ${ }^{50}$.

Plus largement, les documents relatifs aux waqf-s semblaient souvent avoir valeur probatoire, qu'ils proviennent du dīwān d'un ancien cadi ou qu'ils soient apportés par des particuliers, même dans le cas de fondations récentes. Al-Khașsāa évoque le cas où une propriété agricole $\left(\right.$ day $\left.^{\prime} a\right)$ serait immobilisée, tout en demeurant en possession du fondateur et ancien propriétaire. Les bénéficiaires du waqf risquaient de réclamer la première récolte après la transformation de la terre, tandis que le fondateur prétendait n'avoir constitué celle-ci en waqf que depuis un mois - durée inférieure à la croissance et à la maturation des semis -, et par conséquent avoir le droit de récolter ce qu'il avait semé en tant que propriétaire. En ce cas, il revenait au cadi de se reporter à la date de l'éventuel document de fondation et de la comparer à celle de la récolte : si celle-ci avait eu le temps de pousser depuis la fondation, elle appartenait aux bénéficiaires du waqf, à moins que le fondateur n'affirme l'avoir lui-même semée sur ses dépenses personnelles ${ }^{51}$. La date portée sur le document faisait en l'occurrence office de preuve décisive. L'exemple d'un procès orchestré par 'Īsā b. Abān (cadi de Baṣra de 211/826 à 220/835) montre également la valeur probatoire de documents apportés par un plaideur : un personnage appelé Ibn 'A' isha fut traîné en justice par un groupe de Hāšimites qui l'accusaient de profiter illégalement des bénéfices d'un waqf. Pour sa défense, l'homme affirma que les cadis précédents lui en avaient toujours attribué une part. Le cadi 'Īsā b. Abān lui demanda d'apporter la preuve écrite (hujja) d'au moins un de leurs jugements en sa faveur, auquel cas il le compterait à son tour parmi les bénéficiaires du waqf $f^{52}$. Cela s'appliquait peut-être aussi à d'autres types d'affaires : à la fin $\mathrm{du}^{\mathrm{e}} / \mathrm{VIII}^{\mathrm{e}}$ siècle, le cadi égyptien al-Bakrī annula un jugement de son prédécesseur en déchirant la copie détenue par les bénéficiaires $^{53}$. Il s'agissait probablement de les empêcher d'apporter plus tard la preuve de ce jugement - preuve dont la trace écrite demeurait un élément indispensable.

${ }^{48}$ Ibid., p. 114, 159. Cf. E. TYAN, Le notariat et le régime de la preuve par écrit dans la pratique du droit musulman, Beyrouth, 1945, p. 86-87. Tyan relevait déjà l'usage de l'istihsāan pour justifier l'acceptation de la preuve écrite, mais sans en dater l'apparition.

${ }^{49}$ Ibid., p. 114.

${ }^{50}$ Ibid., p. 115.

${ }^{51}$ Ibid., p. 224.

${ }^{52}$ Al-ȘAYMARī, Akhbār Abī Hanīfa, Beyrouth, 1985, p. 152. L'homme en fut incapable et fut donc exclu du partage.

${ }^{53}$ AL-KINDĪ, Akhbār qud̄āt Miṣr, p. 414. 
La préservation des waqf-s et le respect des clauses de distribution de leurs revenus nécessitait un recours régulier à leurs actes de fondation. Même en dehors de tout litige, il revenait aux cadis de surveiller leur gestion. C'est pourquoi il serait inconcevable que ces actes n'aient pas été soigneusement conservés par l'administration judiciaire, soit dans les archives des anciens cadis, soit intégrés à chaque passation de pouvoir au dīwān du cadi entrant. Les prescriptions d'al-Khașșâf concernant la gestion des waqf-s n'ont de sens que dans la mesure où une partie au moins des archives des anciens cadis faisait l'objet d'une conservation pérenne.

Bien que de nombreuses questions demeurent sans réponse, ces quelques éléments conduisent à relativiser les conclusions de Hallaq. Dans l'Irak et l'Égypte abbassides, la transmission du dīwān d'un cadi à son successeur était une étape essentielle de la transition judiciaire. Il semble qu'en règle générale, le cadi entrant prenait physiquement possession des archives avant d'en faire dresser un inventaire, qui garantissait plus tard l'authenticité des documents et fournissait un outil de référence aisément utilisable. Rien ne permet de penser que les cadis révoqués conservaient généralement leurs archives. Le nouveau cadi n'en assurait pas lui-même la conservation intégrale mais les confiait à des hommes de confiance, répartissant peut-être entre plusieurs les $d \bar{\imath} w \bar{a} n$-s de chacun de ses prédécesseurs. Le plus souvent, les documents établis par les anciens cadis ne pouvaient servir de preuve. Mais ils n'en étaient pas pour autant dénués de valeur : ils demeuraient la trace visible des activités judiciaires passées et, en tant que tels, permettaient de présumer du bien-fondé de leur contenu. Ils facilitaient la tâche des cadis entrants qui leur accordaient visiblement - bien que les juristes n'emploient pas ce concept - le statut de présomption. Peut-être, aussi, les cadis intégraient-il des pièces antérieures à leurs propres $d \bar{l} w \bar{a} n$-s. Contrairement à la théorie couramment admise par les fuqahä', certains documents - tout particulièrement les actes de fondations en waqf-s - pouvaient se voir reconnus une valeur probatoire lorsque la production de toute preuve légale était impossible. Ceci explique que les actes de waqf-s fassent partie des principaux documents ayant survécu pour l'époque médiévale. En revanche, la simple valeur de présomption accordée aux autres documents du dīwān n'encouragea probablement pas à leur conservation minutieuse sur le long terme, au gré des accidents de l'histoire. 